\title{
Spontaneous tumor lysis syndrome in adrenal adenocarcinoma: a case report and review of the literature
}

\author{
Mahan Shafie ${ }^{1,2}$, Alireza Teymouri ${ }^{1}$, Samaneh Parsa ${ }^{3 *}$, Ali Sadeghian $^{2,4}$ and Narjes Zarei Jalalabadi ${ }^{3}$
}

\begin{abstract}
Background: Tumor lysis syndrome is an oncologic emergency that classically occurs following cancer therapy, although spontaneous tumor lysis syndrome can also occur in malignancies, albeit rarely. Spontaneous tumor lysis syndrome has previously been reported in some hematologic malignancies, but it rarely happens in solid tumors and seems to be associated with a higher mortality rate. This is the first case of adrenal adenocarcinoma that developed spontaneous tumor lysis syndrome.

Case presentation: We present a rare case of spontaneous tumor lysis syndrome occurring in a patient previously diagnosed with adrenal adenocarcinoma. The patient was a 64-year-old Persian man with abdominal pain, hypersomnia, and fatigue who was previously diagnosed with right adrenocortical carcinoma and had undergone right adrenalectomy with regional lymph nodes resection 5 months previously. On physical examination, the patient had abdominal distension and mild tenderness at the right upper quadrant. Pitting edema was detected bilaterally in the lower extremities. Initial imaging revealed multiple and large lesions suggestive of liver metastases. The laboratory data showed hyperkalemia, hyperuricemia, hyperphosphatemia, and elevated serum creatinine level indicative of spontaneous tumor lysis syndrome in the patient. Despite immediate and intensive care with antibiotics, hydration, treatment with a hypouricemic agent, and renal replacement therapy, the patient ultimately died from multiorgan failure.

Conclusions: Tumor lysis syndrome in solid tumors has high mortality. Patients susceptible to spontaneous tumor lysis syndrome must receive aggressive treatment immediately, which is crucial for preventing morbidity and mortality. Spontaneous tumor lysis syndrome may be underdiagnosed, and a high degree of clinical suspicion is needed to make the diagnosis and proceed with required interventions. Therefore, clinicians should be aware of this rare phenomenon.
\end{abstract}

Keywords: Spontaneous tumor lysis syndrome, Adrenal adenocarcinoma, Solid tumor

\section{Background}

Tumor lysis syndrome (TLS) is an oncologic emergency that results from the release of intracellular contents including potassium, phosphate, and nucleic acids,

*Correspondence: s-parsa@sina.tums.ac.ir

${ }^{3}$ Department of Internal Medicine, Imam Khomeini Hospital Complex, Keshavarz Boulevard, Post code: 1419733141 Tehran, Iran

Full list of author information is available at the end of the article proteins, and other metabolites into the bloodstream secondary to the rapid breakdown of tumor cells. This condition may lead to acute kidney injury, cardiac arrhythmias, seizures, and ultimately death [1]. Although TLS classically occurs following cancer therapy (chemotherapy, radiotherapy, immunotherapy, or other treatments), spontaneous TLS (STLS) can occur rarely in malignancies. STLS has previously been reported in some hematologic malignancies but is thought to be extremely rare original author(s) and the source, provide a link to the Creative Commons licence, and indicate if changes were made. The images or other third party material in this article are included in the article's Creative Commons licence, unless indicated otherwise in a credit line to the material. If material is not included in the article's Creative Commons licence and your intended use is not permitted by statutory regulation or exceeds the permitted use, you will need to obtain permission directly from the copyright holder. To view a copy of this licence, visit http://creativecommons.org/licenses/by/4.0/. The Creative Commons Public Domain Dedication waiver (http://creativeco mmons.org/publicdomain/zero/1.0/) applies to the data made available in this article, unless otherwise stated in a credit line to the data. 
in solid tumors [2]. Moreover, it is estimated that STLS in solid tumors is associated with more mortality and has a worse prognosis than in hematologic malignancies [3]. In our case, a patient with adrenal adenocarcinoma and subsequent adrenalectomy presented with nonspecific symptoms, confusion, abdominal pain, and electrolyte impairments, highly suggestive of spontaneous tumor lysis. To the best of our knowledge, this is the first case of adrenal adenocarcinoma that developed STLS.

\section{Case presentation}

A 64-year-old Persian man presented with abdominal pain. He was confused and affected by hypersomnia and fatigue. The patient also had retrosternal chest pain, exertional dyspnea, nausea, vomiting, and constipation. He was previously diagnosed with right adrenocortical carcinoma and had undergone right adrenalectomy with regional lymph nodes resection 5 months previously; the histopathology revealed adrenal cortical carcinoma with a score of 6 based on Weiss criteria, and immunohistochemistry was positive for synaptophysin, inhibin, and CKAE1/AE3 (Additional file 1: Fig. S1, Additional file 2: Fig. S2, Additional file 3: Fig. S3). He had a history of hypertension, and amlodipine was his only hypertensive medication. For constipation he had received lactulose, and oxycodone was prescribed for pain relief. The patient was a heavy cigarette smoker and had been using opium for more than 20 years. At admission, the patient had a blood pressure of $120 / 76 \mathrm{mmHg}$ and a heart rate of $107 \mathrm{bpm}$. His respiratory rate was 20 breaths per minute. A body temperature of $36.7^{\circ} \mathrm{C}$ and $\mathrm{SPO}_{2}$ of $93 \%$ were also recorded. On physical examination, the patient had abdominal distension and mild tenderness at the right upper quadrant. Pitting edema was detected bilaterally in the lower extremities. Other physical examinations were insignificant. Chest pain and dyspnea prompted echocardiography (ECG), which was unremarkable with estimated ejection fraction of $55 \%$ and no pericardial effusion. In addition to abdominal pain, nausea, and vomiting, the patient did not have defecation and gas passage for two consecutive days. To evaluate possible gastrointestinal obstruction, consultation with the surgery department and abdominopelvic $\mathrm{x}$-ray were performed. Accordingly, the possibility of obstruction was ruled out. Abdominopelvic computed tomography $(\mathrm{CT})$ revealed multiple and large lesions suggestive of liver metastases. In initial laboratory results, hyperkalemia (potassium $6 \mathrm{mg} / \mathrm{dl}$ ), hyperuricemia (uric acid $16.4 \mathrm{mg}$ / $\mathrm{dl}$ ), hyperphosphatemia (phosphorus $6.9 \mathrm{mg} / \mathrm{dl}$ ), and hypocalcemia (calcium $7.8 \mathrm{mg} / \mathrm{dl}$ ), with elevated serum creatinine level (creatinine $3.8 \mathrm{mg} / \mathrm{dl}$ ), were detected. White blood cell count of $26.58 \times 10^{9} / \mathrm{L}, \mathrm{C}$-reactive protein (CRP) of $45 \mathrm{mg} / \mathrm{L}$, and alkaline phosphatase (ALP) of $1144 \mathrm{IU} / \mathrm{L}$ were indicative of an inflammatory process (Table 1). In urine analysis, significant amounts of blood and protein were found. Due to these electrolyte disturbances (hyperkalemia, hyperuricemia, and hyperphosphatemia), which fulfilled Cairo-Bishop criteria, tumor lysis syndrome was considered as an etiology of acute kidney injury for this patient, and since he had not ever received chemotherapy or radiotherapy, the patient was diagnosed as having spontaneous tumor lysis syndrome (TLS) and treated accordingly. Hydration with crystalloid intravenous fluids such as normal saline at a rate of $200 \mathrm{ml} /$ hour was started as initial management to expand the intracellular volume and increase renal excretion of uric acid and other metabolites. A loop diuretic was used as add-on therapy to prevent volume overload and to wash out obstructing uric acid crystals. After ensuring a sufficient level of G6PD in the patient, rasburicase was administered to convert uric acid to more soluble and easily excreted compounds. Hyperkalemia, a life-threatening abnormality in TLS, was managed with sodium polystyrene sulfonate as a potassium-lowering agent. Insulin plus hypertonic glucose was also prescribed as a temporizing measure. In addition to massive hydration and hypouricemic agents, we considered restriction of dietary phosphate intake and administration of a phosphate binder such as sevelamer carbonate, to treat hyperphosphatemia. Blood and urine cultures were drawn, and piperacillin-tazobactam was administrated to treat possible sepsis. Due to volume overload signs (i.e., pulmonary congestion and peripheral edema), severe oliguria, and blunted consciousness during hospitalization, the patient underwent renal replacement therapy with hemodialysis on three consecutive days. Despite immediate and intensive care with hydration, treatment with a hypouricemic agent, and renal replacement therapy, the patient ultimately passed away from cardiopulmonary failure.

\section{Discussion and conclusion}

We present a fatal case of spontaneous tumor lysis as a complication of metastatic adrenal adenocarcinoma. To our knowledge, this is the first case presentation reporting STLS in adrenal adenocarcinoma. TLS is an oncologic emergency that usually occurs after initiation of treatment in hematologic malignancies. It is rarely reported in solid tumors and may occur occasionally in solid tumors with a massive burden after receiving treatment [2]. The diagnosis of TLS is based on Cairo-Bishop criteria [4], and at least two of the following metabolic abnormalities must be present: potassium $\geq 6 \mathrm{mmol} / \mathrm{L}$ or $25 \%$ increase from baseline; phosphorus $\geq 4.6 \mathrm{mg} / \mathrm{dl}$ or $25 \%$ increase from baseline; calcium $\leq 7.0 \mathrm{mg} / \mathrm{dl}$ or $25 \%$ decrease from baseline; uric acid $\geq 8.0 \mathrm{mg} / \mathrm{dl}$ or $25 \%$ 
Table 1 Patient's laboratory data during hospitalization

\begin{tabular}{|c|c|c|c|c|c|c|c|c|}
\hline & On admission & Day 1 & Day 2 & Day 3 & Day 4 & Day 5 & Day 6 & Day 7 \\
\hline $\mathrm{Na}(\mathrm{mEq} / \mathrm{l})$ & 136 & 139 & 133 & 132 & 131 & 132 & 137 & 137 \\
\hline $\mathrm{K}(\mathrm{mEq} / \mathrm{l})$ & 6.0 & 5.3 & 3.7 & 3.2 & 3.1 & 4.5 & 5.1 & 6.0 \\
\hline $\mathrm{BS}(\mathrm{mg} / \mathrm{dl})$ & 55 & 71 & - & - & 57 & - & 55 & 42 \\
\hline Urea (mg/dl) & 189 & 243 & 188 & 196 & 207 & 202 & 207 & 182 \\
\hline $\mathrm{Cr}(\mathrm{mg} / \mathrm{dl})$ & 3.8 & 4.0 & 3.6 & 3.7 & 4.4 & 4.4 & 4.3 & 4.6 \\
\hline $\mathrm{Ca}(\mathrm{mg} / \mathrm{dl})$ & 7.8 & 7.2 & 6.7 & 6.3 & 7.1 & 7.1 & 7.2 & 7.2 \\
\hline$P(\mathrm{mg} / \mathrm{dl})$ & 6.9 & 8.5 & 7.2 & 7.9 & 8.6 & 5.7 & 8.5 & - \\
\hline $\mathrm{Mg}(\mathrm{mg} / \mathrm{dl})$ & 3.1 & 3.5 & 3.0 & 2.3 & 2.6 & 2.7 & 2.8 & 2.6 \\
\hline Uric acid (mg/dl) & $16.4-$ & 4.0 & 7.1 & 2.5 & 2.0 & 1.2 & - & 2.7 \\
\hline CPK (mg/dl) & - & 174 & - & - & - & - & 2.6 & - \\
\hline $\mathrm{LDH}(\mathrm{mg} / \mathrm{dl})$ & - & 8223 & - & - & - & - & - & - \\
\hline WBC (count) & 26,580 & - & - & - & 23,500 & 25,960 & - & 20,800 \\
\hline $\mathrm{Hb}(\%)$ & 9.8 & - & - & - & 10.7 & 9.7 & - & 10 \\
\hline Platelet (count) & 486,000 & - & - & - & 385,000 & 403,000 & - & 341,000 \\
\hline PT (seconds) & 15.3 & - & 17 & - & - & - & - & - \\
\hline PTT (seconds) & 32 & - & 37 & - & - & - & - & - \\
\hline INR & 1.17 & - & 1.3 & - & - & - & - & - \\
\hline AST (mg/dl) & 360 & - & - & - & - & - & - & - \\
\hline $\operatorname{ALT}(\mathrm{mg} / \mathrm{dl})$ & 44 & - & - & - & - & - & - & - \\
\hline $\mathrm{ALP}(\mathrm{mg} / \mathrm{dl})$ & 1144 & - & - & - & - & - & - & - \\
\hline Bilirubin total (mg/dl) & 0.8 & - & - & - & - & - & - & - \\
\hline CRP $(\mathrm{mg} / \mathrm{dl})$ & 45 & - & - & - & - & - & - & - \\
\hline Albumin (g/dl) & - & - & 2.6 & - & - & 2.1 & - & - \\
\hline
\end{tabular}

WBC, white blood cells; $\mathrm{Hb}$, hemoglobin; CRP, C-reactive protein; AST, aspartate aminotransferase; ALT, alanine aminotransferase; ALP, alkaline phosphatase; $\mathrm{LDH}$, lactate dehydrogenase; CPK, creatine phosphokinase; BS, blood sugar; Cr, creatinine; PT, prothrombin time; PTT, partial thromboplastin time; INR, international normalized ratio

increase from baseline. Spontaneous TLS (STLS) is the development of TLS in a patient with malignancy who had not received cancer-specific therapy. STLS rarely happens in solid tumors, and it has been suggested STLS is associated with a high mortality rate according to previous studies [3].

The etiology and risk factors of STLS remain unclear and controversial in solid tumors. Large bulky tumors are considered as risk factors due to insufficient blood flow. However, our patient had underwent adrenalectomy and regional lymph node dissection. In addition, based on previous case reports, multiple liver metastases, as in our case, seemed to contribute to the development of STLS in patients with solid malignancies [5]. A systemic inflammatory syndrome may occur due to necrosis in a cancerous bulk, and resultant infection in advanced metastatic disease, which is also associated with STLS development [6, 7]. Other risk factors for development of STLS in patients with solid tumors include old age, high LDH plasma level, WBC count, and past medical history of renal impairment [5, 8].

Patients with malignancy, who are at risk of developing TLS, should receive immediate and intensive treatment due to a high mortality rate associated with this condition. Cardiac monitoring and urine output measurements should be performed. Blood electrolytes, uric acid, and creatinine should be checked every 4-6 h. Hyperkalemia can lead to cardiac dysrhythmia in these patients and must be taken into consideration, and calcium gluconate should be administrated. Other potassium-lowering strategies such as glucose/insulin infusion, beta-agonist inhalation, and oral potassium-binding agents may also be used. Calcium treatment should be avoided in asymptomatic hypocalcemia due to calcium phosphate formation in the kidneys and possible renal complications. Hypouricemic agents (allopurinol and rasburicase) can be used to reduce uric acid level. Although allopurinol inhibits formation of uric acid crystals, it is not effective in the treatment of acute TLS. On the other hand, rasburicase, with a dosage of $0.2 \mathrm{mg} / \mathrm{kg} /$ day, reduces the uric acid level quickly. Despite all these supportive and pharmacological treatment, some patients with the following indications require hemodialysis: severe oliguria or anuria, persistent hyperkalemia, hyperphosphatemiainduced symptomatic hypocalcemia, and calcium phosphate product $\geq 70 \mathrm{mg} / \mathrm{dl}$ [9]. TLS management 
comprises immediate initiation of supportive care and identification of high-risk patients. Aggressive IV hydration and the diuresis of the patient are of primary importance. Monitoring and treatment of any disturbance of electrolytes is considered to be vital in this condition.

Our literature review revealed 63 cases with solid tumor reporting development of STLS (Additional file 4: Table S1). The mean age of cases at time of developing STLS was 53 years, and 34 of these cases were male. This literature review showed that various tumors may develop STLS; however, the most commonly reported cancers associated with STSL were lung, colorectal, and melanoma. Almost all of them had metastases that involved different sites. Liver metastases were reported in 41 out of 63 cases (65\%). Also, 18 (29\%) cases were reported to have lung involvement. As discussed in previous reports, it seems that metastasis, particularly liver metastasis, may predispose patients to develop STLS. Impaired uric acid metabolism due to hepatic dysfunction following the metastases and spreading of the tumor may be an explanation for these findings [10]. The most common initial symptoms of abdominal pain, discomfort, and distention were reported in about $55 \%$ of cases. The mortality rate of these cases was 69\% (44 cases died out of 63 total cases), which seems to be higher than in hematological malignancies. These data confirm the theory that STLS has worse prognosis in solid tumors than hematological malignancies. Hence, due to the poor prognosis of TLS in malignancies, particularly STLS in solid tumors, it is essential to keep in mind that TLS can occur spontaneously, and it is important to initiate immediate treatment when any suspicious symptoms are noticed.

In conclusion, TLS in solid tumors has a high mortality. Patients susceptible to STLS must receive aggressive treatment immediately, which is crucial for preventing morbidity and mortality. STLS may be underdiagnosed, and a high degree of clinical suspicion is needed to make the diagnosis and proceed with required interventions. Therefore, clinicians should be aware of this rare phenomenon.

\section{Abbreviations}

TLS: Tumor lysis syndrome; STLS: Spontaneous tumor lysis syndrome.

\section{Supplementary Information}

The online version contains supplementary material available at https://doi. org/10.1186/s13256-022-03263-4.

Additional file 1: Figure S1. T1-weighted MRI showing a metastasis of the adrenocortical carcinoma.

Additional file 2: Figure S2. T1-weighted MRI showing right adrenocortical carcinoma tumor.
Additional file 3: Figure S3. T1-weighted MRI showing a metastasis of the adrenocortical carcinoma.

Additional file 4: Table S1. Published cases of spontaneous tumor lysis syndrome in solid tumors.

Acknowledgements

Not applicable.

\section{Authors' contributions}

MS contributed to developing the research idea and composing and revising the manuscript. AT contributed to composing and revising the manuscript. SP contributed to developing the research idea and revising the manuscript. AS contributed to composing and revising the manuscript. NZJ contributed to composing and revising the manuscript. All authors read and approved the final manuscript.

\section{Funding}

Not applicable.

\section{Availability of data and materials}

Data sharing is not applicable to this article as no datasets were generated or analyzed during the current study. Laboratory data and literature review are included in this published article as supplementary files.

\section{Declarations}

Ethics approval and consent to participate

This study was approved by the research and ethics committee of Tehran University of Medical Sciences. The patient's family gave informed consent to publish this case.

\section{Consent for publication}

Written informed consent was obtained from the patient's next of kin for publication of this case report and any accompanying images. A copy of the written consent is available for review by the Editor-in-Chief of this journal.

\section{Competing interests}

The authors have no conflict of interest to declare.

\section{Author details}

${ }^{1}$ School of Medicine, Tehran University of Medical Sciences, Tehran, Iran. ${ }^{2} \mathrm{Neu}-$ roTRACT Association, Students' Scientific Research Center, Tehran University of Medical Sciences, Tehran, Iran. ${ }^{3}$ Department of Internal Medicine, Imam Khomeini Hospital Complex, Keshavarz Boulevard, Post code: 1419733141 Tehran, Iran. ${ }^{4}$ Students'Scientific Research Center, Tehran University of Medical Sciences, Tehran, Iran.

Received: 20 January 2021 Accepted: 6 January 2022

Published online: 10 February 2022

\section{References}

1. Puri I, Sharma D, Gunturu KS, Ahmed AA. Diagnosis and management of tumor lysis syndrome. J Community Hosp Intern Med Perspect. 2020;10(3):269-72.

2. Howard SC, Jones DP, Pui CH. The tumor lysis syndrome. N Engl J Med. 2011;364(19):1844-54.

3. Sommerhalder D, Takalkar AM, Shackelford R, Peddi P. Spontaneous tumor lysis syndrome in colon cancer: a case report and literature review. Clin Case Rep. 2017;5(12):2121-6. https://doi.org/10.1002/ccr3.1269.

4. Cairo MS, Bishop M. Tumour lysis syndrome: new therapeutic strategies and classification. Br J Haematol. 2004;127(1):3-11.

5. Alan AM, Alan O. A case of spontaneous tumor lysis syndrome in extensive-stage small-cell lung cancer: a rare oncologic emergency. Turk J Emerg Med. 2020;20(3):142-5. https://doi.org/10.4103/2452-2473. 290061.eCollection2020Jul-Sep. 
6. Mirrakhimov AE, Ali AM, Khan M, Barbaryan A. Tumor lysis syndrome in solid tumors: an up to date review of the literature. Rare Tumors. 2014;6(2):13.

7. Soares M, Feres GA, Salluh JI. Systemic inflammatory response syndrome and multiple organ dysfunction in patients with acute tumor lysis syndrome. Clinics (Sao Paulo). 2009;64(5):479-81. https://doi.org/10.1590/ s1807-59322009000500016.

8. Hochberg J, Cairo MS. Tumor lysis syndrome: current perspective. Haematologica. 2008;93(1):9-13. https://doi.org/10.3324/haematol.12327.

9. Jones GL, Will A, Jackson GH, Webb NJA, Rule S. Guidelines for the management of tumour lysis syndrome in adults and children with haematological malignancies on behalf of the British Committee for Standards in Haematology. British Journal of Haematology. 2015;169(5):661-71.

10. Gemici C. Tumour lysis syndrome in solid tumours. Clin Oncol. 2006;18(10):773-80.

\section{Publisher's Note}

Springer Nature remains neutral with regard to jurisdictional claims in published maps and institutional affiliations.

- fast, convenient online submission

- thorough peer review by experienced researchers in your field

- rapid publication on acceptance

- support for research data, including large and complex data types

- gold Open Access which fosters wider collaboration and increased citations

- maximum visibility for your research: over $100 \mathrm{M}$ website views per year

At BMC, research is always in progress.

Learn more biomedcentral.com/submissions 